\title{
Targeting Endothelial Adhesion Molecule Transcription for Treatment of Inflammatory Disease: A Proof-of-Concept Study
}

\author{
Liam M. Ashander, Binoy Appukuttan, Yuefang Ma, \\ Dione Gardner-Stephen, and Justine R. Smith \\ Eye \& Vision Health, Flinders University School of Medicine, Flinders Medical Centre, Flinders Drive, Bedford Park, SA 5042, Australia \\ Correspondence should be addressed to Justine R. Smith; justine.smith@flinders.edu.au
}

Received 4 December 2015; Accepted 18 April 2016

Academic Editor: Ronald Gladue

Copyright (c) 2016 Liam M. Ashander et al. This is an open access article distributed under the Creative Commons Attribution License, which permits unrestricted use, distribution, and reproduction in any medium, provided the original work is properly cited.

\begin{abstract}
Targeting the endothelial adhesion molecules that control leukocyte trafficking into a tissue has been explored as a biological therapy for inflammatory diseases. However, these molecules also participate in leukocyte migration for immune surveillance, and inhibiting the physiological level of an adhesion molecule might promote infection or malignancy. We explored the concept of targeting endothelial adhesion molecule transcription during inflammation in a human system. Intercellular adhesion molecule 1 (ICAM-1) mediates leukocyte migration across the retinal endothelium in noninfectious posterior uveitis. We observed an increase in the transcription factor, nuclear factor of kappa light polypeptide gene enhancer in B-cells 1 (NF- $\kappa$ B1), in parallel with ICAM-1, in human retinal endothelial cells treated with tumor necrosis factor-alpha (TNF- $\alpha$ ), and identified putative binding sites for NF- $\kappa$ B1 within the ICAM-1 regulatory region. We targeted induced NF- $\kappa \mathrm{B} 1$ expression in endothelial cells with small interfering (si)RNA. Knockdown of NF- $\kappa$ B1 significantly decreased cell surface expression of ICAM-1 protein induced by TNF- $\alpha$ but did not reduce constitutive ICAM-1 expression. Consistently, NF- $\kappa$ B1 knockdown significantly reduced leukocyte binding to cell monolayers in the presence of TNF- $\alpha$ but did not impact baseline binding. Findings of this proof-of-concept study indicate that induced transcription of endothelial adhesion molecules might be targeted therapeutically for inflammatory disease in humans.
\end{abstract}

\section{Introduction}

Inflammatory diseases are characterized by the accumulation of leukocytes within one or more body tissues. The ingress of leukocytes into a tissue from the blood stream is directed by the adhesion molecules and chemokines that are expressed on the vascular endothelial lining of blood vessels coursing through that tissue [1]. By recognizing receptors on the leukocyte surface, endothelial proteins coordinate the consecutive stages of leukocyte migration: rolling; firm adhesion; spreading and crawling; and diapedesis. This interaction has specificity, since endothelial adhesion molecules and chemokines differ between vascular beds and across immunopathologies $[2,3]$.

Targeting the endothelial adhesion molecules that control leukocyte trafficking into a tissue during inflammation has appeal as a biological treatment for inflammatory disease [4]. However, a major challenge in the therapeutic application of any biologic drug is the need to permit normal physiological processes, while simultaneously targeting disease mechanisms. Adhesion molecules also participate in leukocyte migration for immune surveillance, which is the process by which leukocytes patrol the organs of the body, to determine the need for immune responses to threats such as tissue injury, infectious pathogens, and tumor cells $[5,6]$. Inhibiting this physiological leukocyte activity could place the patient with inflammatory disease at high risk of infection and/or cancer. Indeed, clinical use of the biologic drug, natalizumab, which targets adhesion molecule, $\alpha 4$-integrin (CD49D), has been linked to the occurrence of melanoma, lymphoma, infections, and progressive multifocal leukoencephalopathy [7].

During inflammation, expression of endothelial adhesion molecules and chemokines increases in response to cytokines and other inflammatory mediators [8]. Induction of these proteins depends on the molecular signal(s) and the endothelial subpopulation, and control may be exercised at 
the level of gene transcription [9]. These observations suggest an innovative therapeutic approach: targeting the induced expression of an endothelial adhesion molecule involved in leukocyte migration might spare constitutive expression and allow physiological functions to continue undisturbed.

Noninfectious posterior uveitis is a group of visionthreatening, retina-based inflammatory diseases mediated by CD4+ helper T cells and myeloid cells [10]. Work from our group and from others has demonstrated a significant role for the immunoglobulin (Ig) superfamily member, intercellular adhesion molecule 1 (ICAM-1, CD54), in interactions between human leukocyte subsets and the retinal vascular endothelium [11, 12]. Master inflammatory cytokine, tumor necrosis factor-alpha (TNF- $\alpha$ ), plays an important role in the progression of noninfectious posterior uveitis. Examination of blood and ocular fluid samples from affected persons with uveitis indicates high levels of TNF- $\alpha$ [13-16], and TNF$\alpha$ blockade is often highly effective in patients with recalcitrant inflammation $[17,18]$. In a proof-of-concept study, we investigated the impact of transcription factor blockade on TNF- $\alpha$-induced-versus constitutive-ICAM-1 expression by human retinal endothelial cells and on the interaction between leukocytes and human retinal endothelium.

\section{Materials and Methods}

2.1. RNA Silencing Reagents, Cytokines, and Antibodies. Nontargeted small interfering (si)RNA (Silencer Select Negative Control Number 1 siRNA, catalogue number 4390843) and siRNA designed to target nuclear factor of kappa light polypeptide gene enhancer in B-cells 1 (NF- $\kappa \mathrm{B} 1$ ) (Silencer Select siRNA, catalogue number 4392420) were purchased from ThermoFisher Scientific-Ambion (Foster City, CA). Lipofectamine RNAiMAX Transfection Reagent was sourced from ThermoFisher Scientific-Invitrogen (Carlsbad, CA). Human recombinant TNF- $\alpha$ was bought from R\&D Systems (Minneapolis, MN). Mouse monoclonal anti-human ICAM-1 antibody (clone LB-2, isotype $\operatorname{IgG}_{2 \mathrm{~b}, \kappa}$ ) and mouse monoclonal IgG (clone 27-35, isotype $\operatorname{IgG}_{2 b, \kappa}$ ) were obtained from BD Pharmingen (San Jose, CA). Alexa Fluor 488conjugated goat anti-mouse $\operatorname{IgG}(\mathrm{H}+\mathrm{L})$ secondary antibody was obtained from ThermoFisher Scientific-Molecular Probes (Eugene, OR).

2.2. Endothelial Cell and Leukocyte Lines. Our method for generating human retinal endothelial cell lines has previously been reported in detail [22]. In brief, endothelial cells were isolated from human cadaver retinae by enzymatic digestion of tissue and selection with magnetic bead-conjugated antihuman CD31 antibody and subsequently expanded by transduction with the mouse recombinant amphotropic retrovirus, LXSN16E6E7 [23]. Work presented in our previous publication shows that these retinal endothelial cell lines retain their endothelial phenotype, including expression of endothelial markers and formation of capillary-like tubes on basement membrane substitute [22]. Human retinal endothelial cells were cultured in MCDB-131 medium (SigmaAldrich, St. Louis, MO) supplemented with $10 \%$ fetal bovine serum (FBS) (HyClone-GE Healthcare Life Sciences, Logan, UT) and endothelial growth factors (EGM-2 SingleQuots supplement, omitting FBS, hydrocortisone, and gentamicin; Clonetics-Lonza, Walkersville, $\mathrm{MD}$ ) at $37^{\circ} \mathrm{C}$ and $5 \% \mathrm{CO}_{2}$. The THP-1 human leukocyte line (American Type Culture Collection, Manassas, VA) was maintained in suspension in RPMI1640 medium (ThermoFisher-GIBCO, Grand Island, NY) supplemented with $10 \%$ FBS and 0.05 mM 2-mercaptoethanol.

2.3. RNA Isolation, Reverse Transcription, and Polymerase Chain Reaction. At the conclusion of retinal endothelial cell manipulations, culture medium was replaced with Buffer RLT (Qiagen, Hilden, Germany) with $0.55 \mathrm{mM} \beta$-mercaptoethanol (Sigma-Aldrich, St. Louis, MO), and cells were frozen at $-80^{\circ} \mathrm{C}$ prior to RNA extraction. Total RNA was extracted using the RNeasy mini kit (Qiagen), according to the manufacturer's instructions and including the optional on-column DNase treatment. RNA concentration was determined by spectrophotometry on the NanoDrop 2000 instrument (ThermoFisher Scientific, Wilmington, DE). Reverse transcription was performed using the iScript Reverse Transcription Supermix for RT-qPCR (Bio-Rad, Hercules, CA), with $250 \mathrm{ng}$ (quantitative real-time polymerase chain reaction (PCR)) or $500 \mathrm{ng}$ (PCR array) of RNA template yielding $20 \mu \mathrm{L}$ cDNA.

Quantitative real-time PCR was performed on the CFX Connect Real-Time PCR Detection System (Bio-Rad) using $2 \mu \mathrm{L}$ of cDNA, $4 \mu \mathrm{L}$ of iQ SYBRGreen Supermix, $1.5 \mu \mathrm{L}$ each of $20 \mu \mathrm{M}$ forward and reverse primers, and $11 \mu \mathrm{L}$ of nucleasefree water for each reaction. Amplification consisted of a precycling hold at $95^{\circ} \mathrm{C}$ for 5 minutes; 40 cycles of denaturation for 30 seconds at $95^{\circ} \mathrm{C}$; annealing for 30 seconds at $60^{\circ} \mathrm{C}$; extension for 30 seconds at $72^{\circ} \mathrm{C}$; and a postextension hold at $75^{\circ} \mathrm{C}$ or $81^{\circ} \mathrm{C}$ for 1 second. A melting curve, consisting of a 1-second hold at every $0.5^{\circ} \mathrm{C}$ between $70^{\circ} \mathrm{C}$ and $95^{\circ} \mathrm{C}$, was generated to confirm that a single PCR product was produced. For each primer set, standard curves were generated with serially diluted PCR product to confirm an efficiency of $90 \%$ or greater. Primer sequences for all transcripts, including reference genes used for normalization, are presented in Table 1.

2.4. Polymerase Chain Reaction Array Profiling and Selection of Transcription Factors. Confluent monolayers of human retinal endothelial cells in modified MCDB-131 medium were treated with TNF- $\alpha(10 \mathrm{ng} / \mathrm{mL})$ or no cytokine $(n=5$ monolayers/condition) for 60 minutes or 24 hours at $37^{\circ} \mathrm{C}$ and $5 \% \mathrm{CO}_{2}$. Total RNA was isolated and reverse-transcribed, as described above. RNA integrity numbers were 9.7 or higher by the 2100 Bioanalyzer (Agilent Technologies, Waldbronn, Germany). Polymerase chain reaction array was performed using the Bio-Rad PrimePCR Transcription Factor (SAB Target List) H96 Assay PCR array and following the manufacturer's instructions exactly, including the use of SsoAdvanced Universal SYBR Green Supermix (Bio-Rad), with $2 \mu \mathrm{L}$ cDNA per reaction. Results for TNF- $\alpha$-treated versus control cells were normalized to the array reference genes-GAPDH, HPRT1, and TBP_-and compared using the "Gene Study" function of CFX Manager software (v.3.1, Bio-Rad). 
TABLE 1: Primer pairs and product sizes for gene transcripts studied in human retinal endothelial cells. References are provided for primer sequences sourced from the literature. For primer pairs designed in-house, products were confirmed by sequencing.

\begin{tabular}{|c|c|c|}
\hline Gene transcript $^{*}$ & Primer pair & Product size (bp) \\
\hline \multirow{2}{*}{ NF- $\kappa$ B1 [19] } & Forward: 5'-CCCAGTGAAGACCACCTCTC-3' & \multirow{2}{*}{132} \\
\hline & Reverse: 5'-CTGAGTTTGCGGAAGGATGT-3' & \\
\hline \multirow{2}{*}{ ICAM-1 [20] } & Forward: $5^{\prime}$-TAAGCCAAGAGGAAGGAGCA-3' & \multirow{2}{*}{282} \\
\hline & Reverse: $5^{\prime}$-CATATCATCAAGGGTTGGGG-3' & \\
\hline \multirow{2}{*}{ ICAM-1 } & Forward: $5^{\prime}$-GGCCTCAGTCAGTGTGA-3' & \multirow{2}{*}{218} \\
\hline & Reverse: $5^{\prime}$-AACCCCATTCAGCGTCA-3' & \\
\hline \multirow{2}{*}{ 18S rRNA } & Forward: 5'-GTAACCCGTTGAACCCCATT-3' & \multirow{2}{*}{151} \\
\hline & Reverse: $5^{\prime}$-CCATCCAATCGGTAGTAGCG-3' & \\
\hline \multirow{2}{*}{ Cyclophilin A } & Forward: $5^{\prime}$-GACCTCTGGAGAGAAAGGATTT-3' & \multirow{2}{*}{355} \\
\hline & Reverse: $5^{\prime}$-GGTGATCTTCTTGCTGGTCTT-3' & \\
\hline
\end{tabular}

${ }^{*} \mathrm{NF}-\kappa \mathrm{B} 1=$ nuclear factor $\kappa$-light-chain-enhancer of activated B-cells 1; ICAM-1 = intercellular adhesion molecule 1; 18S rRNA = 18S ribosomal RNA.

In order to identify one candidate transcription factor for our proof-of-concept study, we required significant increase from baseline expression at both 60 minutes and 24 hours $(p<0.01)$, plus a 1.5 -fold increase in expression at one or both time points. We interrogated the ICAM-1 regulatory sequence for potential binding sites of transcription factors that fulfilled these criteria. We obtained human ICAM-1 transcript sequence from the National Center for Biotechnology Information "Nucleotide" database (i.e., NG_012083.1: Homo sapiens intercellular adhesion molecule 1 (ICAM1), RefSeqGene on chromosome 19), and we defined the regulatory sequence as the $5000 \mathrm{bp}$ of sequence immediately upstream of the ICAM-1 gene transcription start site, plus the first 2 introns ( $3538 \mathrm{bp}$ and $8452 \mathrm{bp}$ ). The regulatory sequence was searched for putative binding sites of human transcription factors using the public JASPAR database [21], with relative profile score threshold set at $80 \%$.

2.5. RNA Silencing. Confluent monolayers of human retinal endothelial cells were transfected with $10 \mathrm{nM} \mathrm{NF-} \kappa \mathrm{B} 1-$ targeted siRNA or nontargeted control siRNA using $1.7 \mu \mathrm{L} /$ mL Lipofectamine RNAiMAX Reagent in modified MCDB131 medium, following the manufacturer's "Forward Transfection" protocol for human umbilical vein endothelial cells. Concentrations, timing, and siRNA were determined in a pilot study (data not shown). After 24 hours, the transfection mixture was replaced with medium only. Monolayers treated with NF- $\kappa$ B1-targeted siRNA or nontargeted siRNA were cultured for an additional 24 hours and subsequently treated with TNF- $\alpha(10 \mathrm{ng} / \mathrm{mL})$ or no cytokine in fresh medium for a final 24 hours.

2.6. Retinal Endothelial ICAM-1 ELISA. The human retinal endothelial ICAM-1 ELISA was adapted from the protocol published by Hartwig et al. [24]. Confluent monolayers of endothelial cells in wells of 96-well plates, transfected with NF- $\kappa$ B1-targeted siRNA or nontargeted control siRNA and treated with TNF- $\alpha$ or no cytokine, were washed twice in phosphate buffered saline (PBS) with 0.1\% Tween-20 (PBS/ Tween-20), fixed in $1 \%$ paraformaldehyde for 30 minutes, and washed in PBS/Tween-20. Following a 30-minute block with $5 \% \mathrm{w} / \mathrm{v}$ skim milk in PBS (blocking solution), monolayers were incubated with anti-human ICAM-1 antibody or mouse monoclonal IgG at $1 \mu \mathrm{g} / \mathrm{mL}$ in blocking solution for 45 minutes at room temperature $(n=8$ monolayers/condition) and washed 5 times with PBS/Tween-20. Subsequently, monolayers were incubated with Alexa Fluor 488-conjugated secondary antibody at $2.5 \mu \mathrm{g} / \mathrm{mL}$ in blocking solution for 30 minutes at room temperature and washed 5 times with PBS/ Tween-20. Finally monolayers were treated with $300 \mathrm{nM} \mathrm{4}{ }^{\prime}, 6-$ diamidino-2-phenylindole-dihydrochloride (DAPI) (SigmaAldrich) in PBS for 5 minutes and washed 3 times in PBS. All treatments after the fixation step were performed on a slowly rotating orbital shaker.

Monolayer fluorescence was determined on the VICTOR X3 Multilabel Plate Reader (Perkin Elmer, Waltham, MA) using 485 excitation and 535 emission (Alexa Fluor 488) filters and 355 excitation and 460 emission (DAPI) filters. Mean background fluorescence was determined from mouse IgGincubated wells for each condition, and this value was subtracted from wells incubated with anti-human ICAM-1 antibody, for the matching condition. Any difference in cell numbers between wells was accounted for by adjusting Alexa Fluor 488 readings for DAPI readings from the same wells.

2.7. Leukocyte-Retinal Endothelial Cell Adhesion Assay. Confluent monolayers of human retinal endothelial cells in wells of 48 -well plates, transfected with NF- $\kappa$ B1-targeted siRNA or nontargeted control siRNA and treated with TNF- $\alpha$ or no cytokine, were incubated with $1 \times 10^{5}$ THP-1 leukocytes suspended in modified MCDB-131 medium ( $n=5$ monolayers/condition) for 20 minutes at $37^{\circ} \mathrm{C}$ and $5 \% \mathrm{CO}_{2}$. After removal of the THP-1 leukocyte suspensions, monolayers were washed 4 times in medium, rotating the plate 90 degrees with each wash, and fixed in $10 \%$ neutral buffered formalin for 10 minutes. The center of each well was photographed under 100x magnification on the IX53 inverted microscope (Olympus, Tokyo, Japan) and with a UC50 color CCD camera (Olympus Soft Imaging Solutions, Muenster, Germany) and cellSens Imaging Software (v.1.8.1, Olympus). The number of 


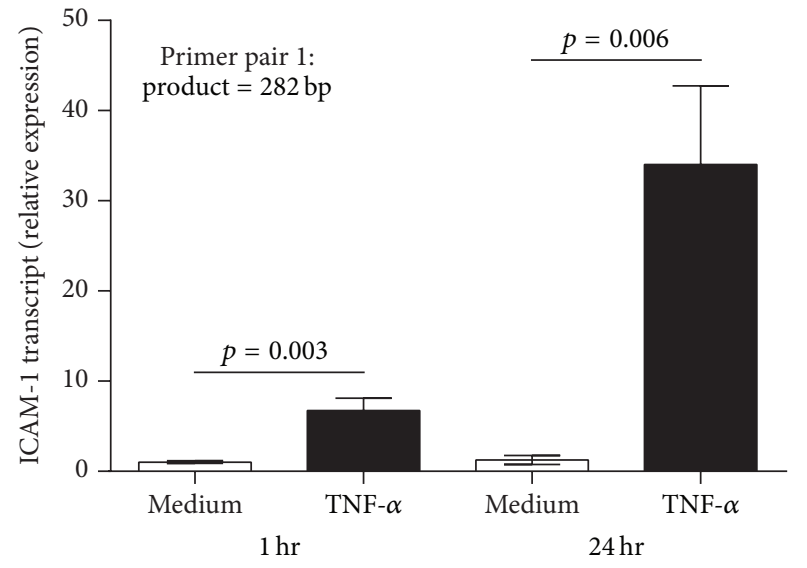

(a)

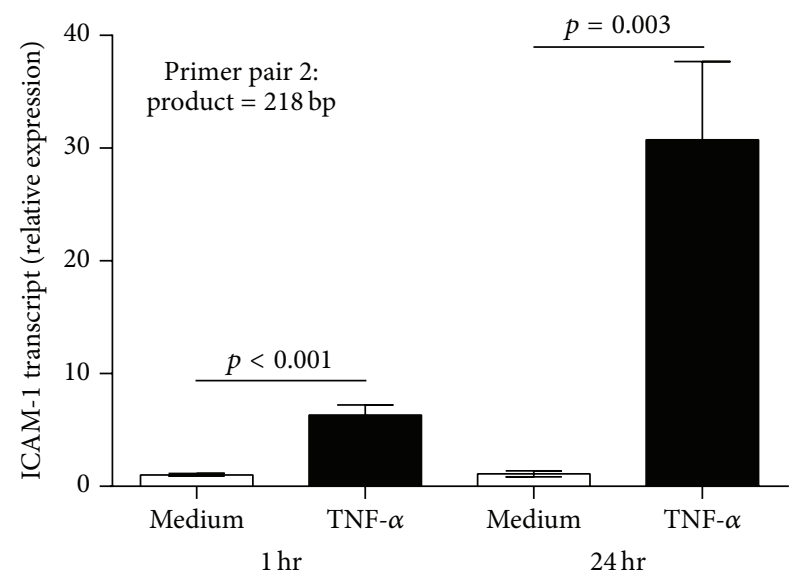

(b)

FIGURE 1: Graphs showing relative ICAM-1 transcript expression in human retinal endothelial cell monolayers, following stimulation with TNF- $\alpha$ for 60 minutes or 24 hours, as determined by qRT-PCR using two ICAM-1 primer pairs. Reference gene was $18 \mathrm{~S}$ rRNA. The same result was obtained when data were normalized to cyclophilin A reference gene. (a) shows the result obtained using ICAM-1 primer pair listed in Table 1 that yields 282 bp product. (b) shows similar result obtained using ICAM-1 primer pair listed in Table 1 that yields 218 bp product. Bars represent mean relative expression, with error bars showing standard error of the mean. $n=5$ monolayers/condition. Data were analyzed by two-tailed Student's $t$-test. Human retinal endothelial RNA samples used for this experiment were used for PCR array profiling study presented in Table 2.

THP-1 leukocytes bound to the human retinal endothelial cell monolayer in each photograph was counted and converted to bound leukocytes $/ \mathrm{mm}$.

2.8. Statistical Analysis. Analysis of the PCR array is described above. For other experiments, data for test and control conditions were compared by two-tailed Student's $t$-tests, using GraphPad Prism v6.04 (GraphPad Software, La Jolla, CA). In all analyses, a significant difference was defined as one yielding a $p$ value less than 0.05 .

\section{Results and Discussion}

Our proof-of-concept study explored the effect of transcription factor blockade on induced-versus constitutiveexpression of an endothelial adhesion molecule in a human uveitis model. Noninfectious posterior uveitis involves the migration of multiple leukocyte subsets across the retinal vascular endothelium. Intercellular adhesion molecule-1 is a key adhesion molecule for leukocyte egress into human retina [11, 12], and TNF- $\alpha$ is a master cytokine regulator of inflammation within human retina [13-16]. We treated human retinal endothelial cells with TNF- $\alpha$ and observed a substantial increase in ICAM-1 transcript expression over 24 hours, beginning within 60 minutes of exposure (Figure 1). This finding indicates that ICAM-1 gene transcription might be targeted to limit induction of ICAM-1 protein expression on retinal endothelial cells and leukocyte-retinal endothelial interactions, without impacting baseline levels of these parameters.
Expression of ICAM-1 is regulated primarily at transcription; activation of the ICAM-1 gene regulatory sequence is cell- and stimulus-specific $[25,26]$. To identify transcription factors involved in retinal endothelial ICAM-1 induction, we profiled TNF- $\alpha$-treated human retinal endothelial cells against cytokine-naïve control cells, using a PCR array that detected 86 well-characterized human transcription factors. In analyzing the results of the PCR array, we focused on transcription factors that increased significantly in parallel with ICAM-1 and demonstrated at least 1.5 -fold increase in expression, following exposure to TNF- $\alpha$. The $5000 \mathrm{bp}$ of sequence immediately upstream of the ICAM-1 gene transcription start site, plus the first 2 introns (3538 bp and $8452 \mathrm{bp}$ ), were searched for putative binding sites of the candidate transcription factors. We employed JASPAR, which is an open-access database of matrix-based nucleotide transcription factor binding profiles [21].

Seven transcription factors were upregulated in human retinal endothelial cells on exposure to TNF- $\alpha$ and had potential binding sites in the ICAM-1 regulatory sequence (Table 2). For the purposes of proving concept, we selected NF- $\kappa$ B1 from the group of 7 candidate transcription factors. This selection was based on the knowledge that NF- $\kappa \mathrm{B} 1$ has been linked to noninfectious posterior uveitis; for example, several groups have reported an association between NF- $\kappa B 1$ polymorphisms and Behçet disease, in which posterior uveitis is classically an occlusive retinal vasculitis $[27,28]$. The JASPAR analysis indicated that the ICAM-1 regulatory region included at least 25 putative binding sites for NF- $\kappa \mathrm{B} 1$ (Table 3 ).

Despite initial enthusiasm for therapeutic manipulation of gene expression, transcription soon came to be regarded as "undruggable" [29]. Potential targets in transcription include 
TABLE 2: Transcription factors with putative binding sites within the regulatory region of intercellular adhesion molecule 1 (ICAM-1) that increase in human retinal endothelial cells treated with tumor necrosis factor-alpha (TNF- $\alpha$ ). For the purposes of this analysis, the ICAM-1 regulatory region was defined as $5000 \mathrm{bp}$ of sequence immediately upstream of the ICAM-1 gene transcription start site and the first 2 introns. The ICAM-1 gene sequence was interrogated in the JASPAR database [21].

\begin{tabular}{lccccc}
\hline \multirow{2}{*}{$\begin{array}{c}\text { Transcription factor } \\
\end{array}$} & \multicolumn{2}{c}{ Induction with TNF- $\alpha$ minutes } & & & \\
& Fold increase & $p$ value & Fold increase & $p$ value & Number of binding sites \\
\hline ETS1 & 1.65 & $<10^{-5}$ & 2.15 & $<10^{-5}$ & 48 \\
EGR1 & 1.36 & $<10^{-5}$ & 2.31 & $<10^{-5}$ & 268 \\
JunB & 2.95 & $<10^{-5}$ & 5.73 & $<10^{-5}$ & 148 \\
IRF1 & 4.77 & $<10^{-5}$ & 5.11 & $<10^{-5}$ & 83 \\
NF- $\kappa$ B1 & 1.24 & $<10^{-3}$ & 2.75 & $<10^{-5}$ & 25 \\
c-Rel & 1.55 & $<10^{-3}$ & 1.38 & $<10^{-4}$ & 101 \\
TGIF1 & 1.31 & $<10^{-4}$ & 1.70 & $<10^{-5}$ & 6 \\
\hline
\end{tabular}

${ }^{*}$ ETS1 = E26 transformation-specific 1; EGR1 = early growth response 1; IRF1 = interferon regulatory factor 1; NF- $\kappa$ B1 = nuclear factor $\kappa$-light-chain-enhancer of activated B-cells 1; TGIF1 = TGFB-induced factor homeobox 1 .

TABLE 3: Sequences and locations of NF- $\kappa$ B1 putative binding sites within the regulatory region of intercellular adhesion molecule 1 (ICAM-1). For the purposes of this study, the ICAM-1 regulatory region was defined as $5000 \mathrm{bp}$ of sequence immediately upstream of the ICAM-1 gene transcription start site and the first 2 introns. Gene sequences were obtained from the JASPAR database [21].

\begin{tabular}{|c|c|c|c|c|}
\hline \multirow{2}{*}{ Sequence } & \multicolumn{4}{|c|}{ Location } \\
\hline & Region & Start & End & Strand \\
\hline AGGAGAATCACCT & \multirow{9}{*}{$\begin{array}{l}5000 \text { bp upstream } \\
\text { of transcription } \\
\text { start site }\end{array}$} & 282 & 294 & -1 \\
\hline AGGTGATTCTCCT & & 282 & 294 & 1 \\
\hline TGGGGTTTCACCA & & 3008 & 3020 & -1 \\
\hline AGGGAGACCCCCA & & 3524 & 3536 & -1 \\
\hline TGGGGGTCTCCCT & & 3524 & 3536 & 1 \\
\hline GGGGGACGCCCCT & & 3874 & 3886 & -1 \\
\hline AGGGGCGTCCCCC & & 3874 & 3886 & 1 \\
\hline GAGGGATGCCCCT & & 4751 & 4763 & -1 \\
\hline AGGGGCATCCCTC & & 4751 & 4763 & 1 \\
\hline TGGGGATTGCCGT & \multirow{2}{*}{ Intron 1 (3538 bp) } & 12 & 24 & 1 \\
\hline GGGGGAATTCCAG & & 584 & 596 & 1 \\
\hline CGGGGTTTCACCA & \multirow{14}{*}{ Intron 2 (8452 bp) } & 1333 & 1345 & -1 \\
\hline TGGTGAAACCCCG & & 1333 & 1345 & 1 \\
\hline CGGGGACTTCCCT & & 3433 & 3445 & -1 \\
\hline AGGGAAGTCCCCG & & 3433 & 3445 & 1 \\
\hline GGGTGGATCACCA & & 3748 & 3760 & -1 \\
\hline TGGTGATCCACCC & & 3748 & 3760 & 1 \\
\hline CGGTGAAACCCCG & & 6035 & 6047 & -1 \\
\hline CGGGGTTTCACCG & & 6035 & 6047 & 1 \\
\hline AGGCGGATCACCT & & 6948 & 6960 & -1 \\
\hline AGGTGATCCGCCT & & 6948 & 6960 & 1 \\
\hline AGGGAGACCCCCA & & 7545 & 7557 & -1 \\
\hline TGGGGGTCTCCCT & & 7545 & 7557 & 1 \\
\hline AGGGGATTCTCCT & & 8082 & 8094 & -1 \\
\hline AGGAGAATCCCCT & & 8082 & 8094 & 1 \\
\hline
\end{tabular}

(1) factors that bind specific DNA sequences to activate or inhibit RNA polymerase II-controlled transcription; (2) coregulators that bind transcription factors to enhance or repress activity; and (3) DNA methylation and histone modifications that direct access of transcription factors to DNAbinding sites. However, the challenges to targeting are significant and include the multiple gene targets of each transcription factor; predominant nuclear location; and large surface areas for DNA- and protein-protein interactions. Over the past 5 years, multiple advances have begun to address these concerns, particularly in oncology, where transcription factor-targeted drugs are now in clinical trials [30]. DNAbinding small molecules, polyamides, and oligonucleotidebased decoys inhibit binding of transcription factors to promoters [29]. Transcription activator-like effectors (TALEs) and zinc finger proteins (ZFPs) provide the opportunity to target specific DNA sequences [31].

We silenced NF- $\kappa$ B1 transcript with siRNA to examine the effect of targeting TNF- $\alpha$-induced ICAM-1 gene transcription in human retinal endothelial cells. ICAM-1 exists as both soluble and membrane-bound forms; only the membranebound form is relevant to the leukocyte-retinal endothelial cell interaction [1]. Thus, we measured the effect of NF- $\kappa \mathrm{B} 1$ silencing on human retinal endothelial cell surface ICAM1 expression, using a cellular ELISA. Knockdown of NF$\kappa \mathrm{B} 1$ significantly decreased TNF- $\alpha$-induced ICAM-1 protein expression but did not reduce constitutive ICAM-1 expression (Figure 2). We next examined the effect of NF- $\kappa \mathrm{B} 1$ silencing on leukocyte-endothelial cell interactions, by quantifying THP-1 leukocyte binding to human retinal endothelial monolayers. Knockdown of NF- $\kappa$ B1 significantly decreased leukocyte binding to human retinal endothelial monolayers that were pretreated with TNF- $\alpha$ but did not reduce binding to nontreated monolayers (Figure 3 ). Thus, NF- $\kappa$ B1-targeted blockade of ICAM-1 gene transcription reduces TNF- $\alpha$ induced inflammatory activities of human retinal endothelial cells that contribute to the development of noninfectious posterior uveitis. Importantly, the blockade does not impact baseline levels of these activities. 


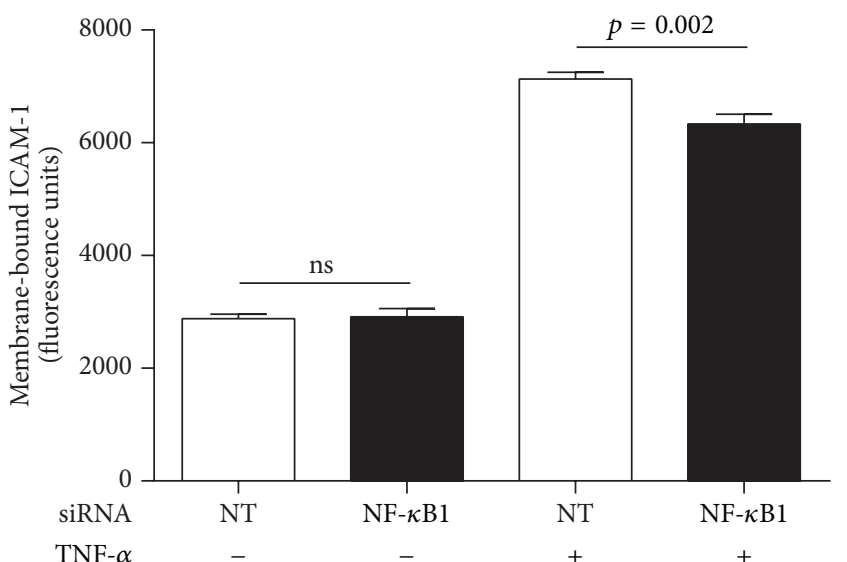

(a)

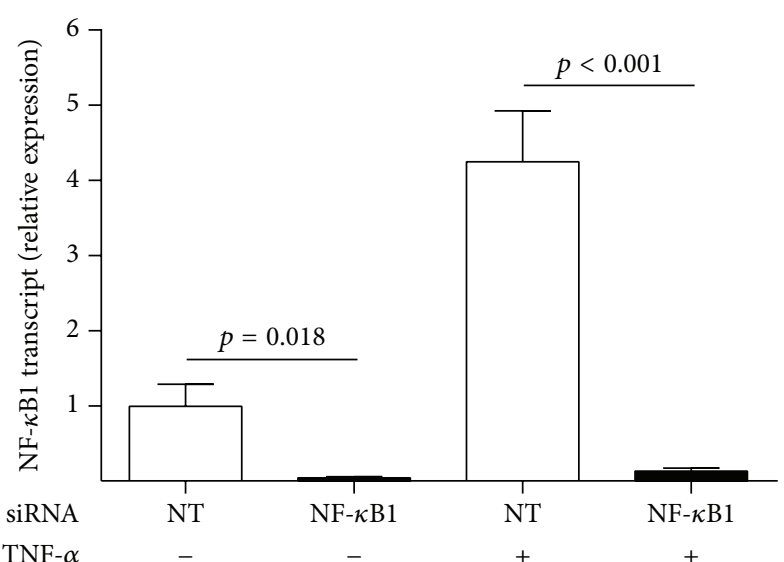

(b)

FIGURE 2: TNF- $\alpha$-induced expression of cellular ICAM-1 is significantly reduced by NF- $\kappa$ B1 silencing, but constitutive expression is not impacted. Human retinal endothelial cell monolayers were transfected with NF- $\kappa$ B1-targeted (NF- $\kappa$ B1) siRNA or nontargeted (NT) control siRNA and treated with TNF- $\alpha$ or no cytokine for 24 hours. Cellular ICAM-1 was fluorescently labeled by indirect immunocytochemistry; fluorescence of endothelial monolayers was read by microplate reader and adjusted for background fluorescence and cell number. (a) Graph showing relative level of ICAM-1 protein on the surface of endothelial cells. Bars represent mean relative level, with error bars showing standard error of the mean. $n=8$ monolayers/condition. Data were analyzed by two-tailed Student's $t$-test. ns $=$ not significant. (b) Graph showing relative NF- $\kappa \mathrm{B} 1$ transcript expression in human retinal endothelial cell monolayers that were transfected in parallel. Reference gene was $18 \mathrm{~S}$ rRNA. Bars represent mean relative expression, with error bars showing standard error of the mean. $n=4$ monolayers/condition. Data were analyzed by two-tailed Student's $t$-test.

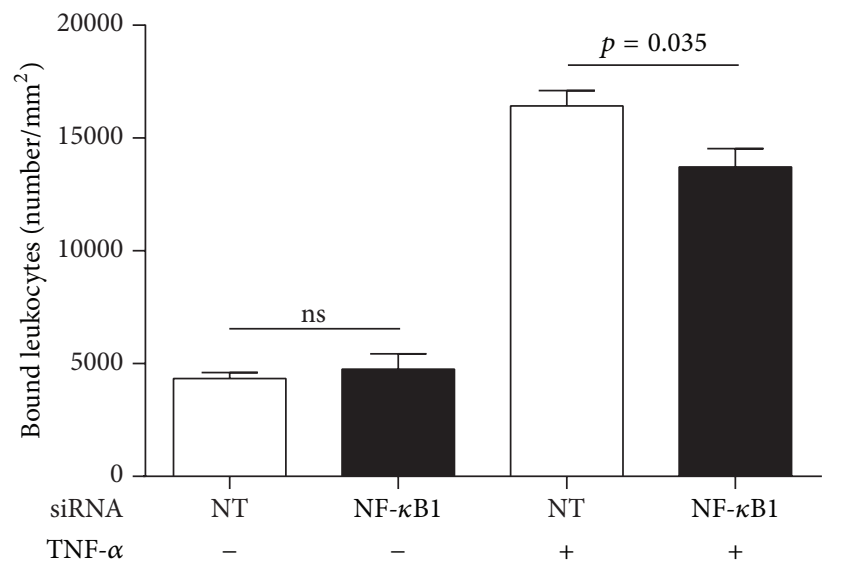

(a)

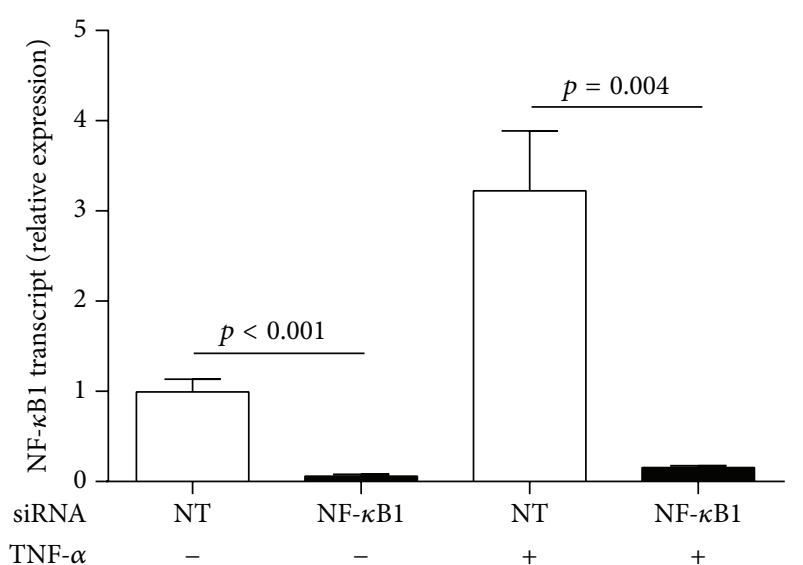

(b)

FIGURE 3: Leukocyte binding to TNF- $\alpha$-stimulated human retinal endothelial monolayers is significantly reduced by NF- $\kappa$ B1 silencing, but binding to untreated monolayers is not impacted. Human retinal endothelial cell monolayers were transfected with NF- $\kappa$ B1-targeted (NF- $\kappa$ B1) siRNA or nontargeted (NT) control siRNA and treated with TNF- $\alpha$ or no cytokine for 24 hours. Endothelial monolayers were incubated with THP-1 leukocytes for 20 minutes $\left(1 \times 10^{5}\right.$ cells $/ 7.5 \mathrm{~mm}^{2}$ monolayer); monolayers were photographed, and the number of leukocytes bound per $\mathrm{mm}^{2}$ was counted. (a) Graph showing leukocytes bound to the surface of endothelial monolayers. Bars represent mean number, with error bars showing standard error of the mean. $n=5$ monolayers/condition. Data were analyzed by two-tailed Student's $t$-test. ns $=$ not significant. (b) Graph showing relative NF- $\kappa$ B1 transcript expression in human retinal endothelial cell monolayers that were transfected in parallel. Reference gene was $18 \mathrm{~S}$ rRNA. Bars represent mean relative expression, with error bars showing standard error of the mean. $n=4$ monolayers/condition. Data were analyzed by two-tailed Student's $t$-test.

\section{Conclusions}

Our research explores a novel treatment paradigm for noninfectious posterior uveitis that also has application to human inflammatory diseases outside the retina. We show the feasibility of targeting induced-but not constitutive-expression on the endothelium of a key adhesion molecule involved in initiation of an inflammatory disease. Sparing constitutive expression is expected to limit the side effects that have complicated the use of other biologic drugs due to the roles of target molecules in physiological leukocyte activity. Targeting transcription of endothelial adhesion molecules might result 
in changes within the cell that lead to other complications. However, current technologies permit highly specific transcription blockade, and recent progress within the pharmaceutical field promises the possibility of local delivery to many organs. Important tasks in translating this therapeutic approach to the clinic are the need to define tissue- and disease-specific adhesion molecules for a given inflammatory condition and consideration of individual utilization of those adhesion molecules in leukocyte-endothelial interactions across multiple human subjects.

\section{Competing Interests}

The authors declare that they have no competing interests regarding the publication of this paper.

\section{Acknowledgments}

The authors wish to thank Keryn A. Williams, Ph.D., and Andrew J. Stempel, B.S. (Flinders University, Adelaide, Australia), for critical review of the paper, and Denise A. Galloway, Ph.D. (Fred Hutchinson Cancer Institute, Seattle, WA), for the gift of the LXSN16E6E7 virus. This work was supported by grants from the National Eye Institute/ National Institutes of Health (R01 EY019875), the Ophthalmic Research Institute of Australia, and the Australian Research Council (FT130101648).

\section{References}

[1] S. Nourshargh and R. Alon, "Leukocyte migration into inflamed tissues," Immunity, vol. 41, no. 5, pp. 694-707, 2014.

[2] D. J. Nolan, M. Ginsberg, E. Israely et al., "Molecular signatures of tissue-specific microvascular endothelial cell heterogeneity in organ maintenance and regeneration," Developmental Cell, vol. 26, no. 2, pp. 204-219, 2013.

[3] D. C. Bullard, "Adhesion molecules in inflammatory diseases: insights from knockout mice," Immunologic Research, vol. 26, no. 1-3, pp. 27-33, 2002.

[4] A. D. Luster, R. Alon, and U. H. von Andrian, "Immune cell migration in inflammation: present and future therapeutic targets," Nature Immunology, vol. 6, no. 12, pp. 1182-1190, 2005.

[5] T. S. Kupper and R. C. Fuhlbrigge, "Immune surveillance in the skin: mechanisms and clinical consequences," Nature Reviews Immunology, vol. 4, no. 3, pp. 211-222, 2004.

[6] S. S. Ousman and P. Kubes, "Immune surveillance in the central nervous system," Nature Neuroscience, vol. 15, no. 8, pp. 10961101, 2012.

[7] T. Derfuss, J. Kuhle, R. Lindberg, and L. Kappos, "Natalizumab therapy for multiple sclerosis," Seminars in Neurology, vol. 33, no. 1, pp. 26-36, 2013.

[8] H. F. Langer and T. Chavakis, "Leukocyte-endothelial interactions in inflammation," Journal of Cellular and Molecular Medicine, vol. 13, no. 7, pp. 1211-1220, 2009.

[9] M. Pate, V. Damarla, D. S. Chi, S. Negi, and G. Krishnaswamy, "Endothelial cell biology. Role in the inflammatory response," Advances in Clinical Chemistry, vol. 52, pp. 109-130, 2010.

[10] M. D. de Smet, S. R. J. Taylor, B. Bodaghi et al., "Understanding uveitis: the impact of research on visual outcomes," Progress in Retinal and Eye Research, vol. 30, no. 6, pp. 452-470, 2011.
[11] A. S. Bharadwaj, L. P. Schewitz-Bowers, L. Wei, R. W. J. Lee, and J. R. Smith, "Intercellular adhesion molecule 1 mediates migration of Thl and Th17 cells across human retinal vascular endothelium," Investigative Ophthalmology and Visual Science, vol. 54, no. 10, pp. 6917-6925, 2013.

[12] Q. Zhang, Y. Jiang, J. J. Toutounchian, C. Soderland, C. R. Yates, and J. J. Steinle, "Insulin-like growth factor binding protein-3 inhibits monocyte adhesion to retinal endothelial cells in high glucose conditions," Molecular Vision, vol. 19, pp. 796-803, 2013.

[13] S. Sugita, Y. Kawazoe, A. Imai, Y. Yamada, S. Horie, and M. Mochizuki, "Inhibition of Th17 differentiation by anti-TNFalpha therapy in uveitis patients with Behçet's disease," Arthritis Research \& Therapy, vol. 14, no. 3, article R99, 2012.

[14] K. Nagata, K. Maruyama, K. Uno et al., "Simultaneous analysis of multiple cytokines in the vitreous of patients with sarcoid uveitis," Investigative Ophthalmology and Visual Science, vol. 53, no. 7, pp. 3827-3833, 2012.

[15] J. J. Kuiper, A. Rothova, P. A. Schellekens, A. Ossewaarde-van Norel, A. C. Bloem, and T. Mutis, "Detection of choroid- and retina-antigen reactive $\mathrm{CD} 8(+)$ and $\mathrm{CD} 4(+) \mathrm{T}$ lymphocytes in the vitreous fluid of patients with birdshot chorioretinopathy," Human Immunology, vol. 75, no. 6, pp. 570-577, 2014.

[16] M. Mesquida, B. Molins, V. Llorenç et al., "Proinflammatory cytokines and C-reactive protein in uveitis associated with Behçet's disease," Mediators of Inflammation, vol. 2014, Article ID 396204, 8 pages, 2014.

[17] E. B. Suhler, J. R. Smith, T. R. Giles et al., "Infliximab therapy for refractory uveitis: 2-year results of a prospective trial," Archives of Ophthalmology, vol. 127, no. 6, pp. 819-822, 2009.

[18] E. B. Suhler, J. R. Smith, M. S. Wertheim et al., "A prospective trial of infliximab therapy for refractory uveitis: preliminary safety and efficacy outcomes," Archives of Ophthalmology, vol. 123, no. 7, pp. 903-912, 2005.

[19] N. A. Rao, M. T. McCalman, P. Moulos et al., "Coactivation of GR and NFKB alters the repertoire of their binding sites and target genes," Genome Research, vol. 21, no. 9, pp. 1404-1416, 2011.

[20] Y. Lu, K. Fukuda, Y. Nakamura, K. Kimura, N. Kumagai, and T. Nishida, "Inhibitory effect of triptolide on chemokine expression induced by proinflammatory cytokines in human corneal fibroblasts," Investigative Ophthalmology \& Visual Science, vol. 46, no. 7, pp. 2346-2352, 2005.

[21] A. Sandelin, W. Alkema, P. Engström, W. W. Wasserman, and B. Lenhard, "JASPAR: an open-access database for eukaryotic transcription factor binding profiles," Nucleic Acids Research, vol. 32, pp. D91-D94, 2004.

[22] A. S. Bharadwaj, B. Appukuttan, P. A. Wilmarth et al., "Role of the retinal vascular endothelial cell in ocular disease," Progress in Retinal and Eye Research, vol. 32, no. 1, pp. 102-180, 2013.

[23] C. L. Kalbert, G. W. Demers, and D. A. Galloway, “The E7 gene of human papillomavirus type 16 is sufficient for immortalization of human epithelial cells," Journal of Virology, vol. 65, no. 1, pp. 473-478, 1991.

[24] W. Hartwig, J. Werner, A. L. Warshaw et al., "Membranebound ICAM-1 is upregulated by trypsin and contributes to leukocyte migration in acute pancreatitis," American Journal of Physiology-Gastrointestinal and Liver Physiology, vol. 287, no. 6, pp. G1194-G1199, 2004.

[25] A. van de Stolpe and P. T. van der Saag, "Intercellular adhesion molecule-1," Journal of Molecular Medicine, vol. 74, no. 1, pp. 1333, 1996. 
[26] C.-C. Chen, "Signal transduction pathways of inflammatory gene expressions and therapeutic implications," Current Pharmaceutical Design, vol. 12, no. 27, pp. 3497-3508, 2006.

[27] B. Yalcin, N. Atakan, and N. Alli, "The functional role of nuclear factor kappa- $\kappa \mathrm{B} 1-94$ ins/del ATTG promotor gene polymorphism in Behçet's disease: an exploratory study," Clinical and Experimental Dermatology, vol. 33, no. 5, pp. 629-633, 2008.

[28] G. Yenmis, T. Oner, C. Cam et al., "Association of NFKB1 and NFKBIA polymorphisms in relation to susceptibility of behçet's disease," Scandinavian Journal of Immunology, vol. 81, no. 1, pp. 81-86, 2015.

[29] C. Yan and P. J. Higgins, "Drugging the undruggable: transcription therapy for cancer," Biochimica et Biophysica Acta (BBA)Reviews on Cancer, vol. 1835, no. 1, pp. 76-85, 2013.

[30] J.E. Yeh, P. A. Toniolo, and D. A. Frank, “Targeting transcription factors: promising new strategies for cancer therapy," Current Opinion in Oncology, vol. 25, no. 6, pp. 652-658, 2013.

[31] J. N. F. Scott, A. P. Kupinski, and J. Boyes, "Targeted genome regulation and modification using transcription activator-like effectors," FEBS Journal, vol. 281, no. 20, pp. 4583-4597, 2014. 


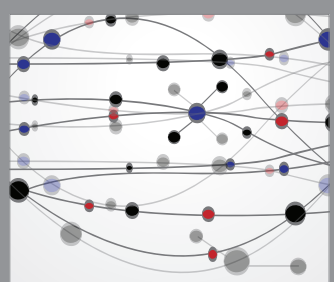

The Scientific World Journal
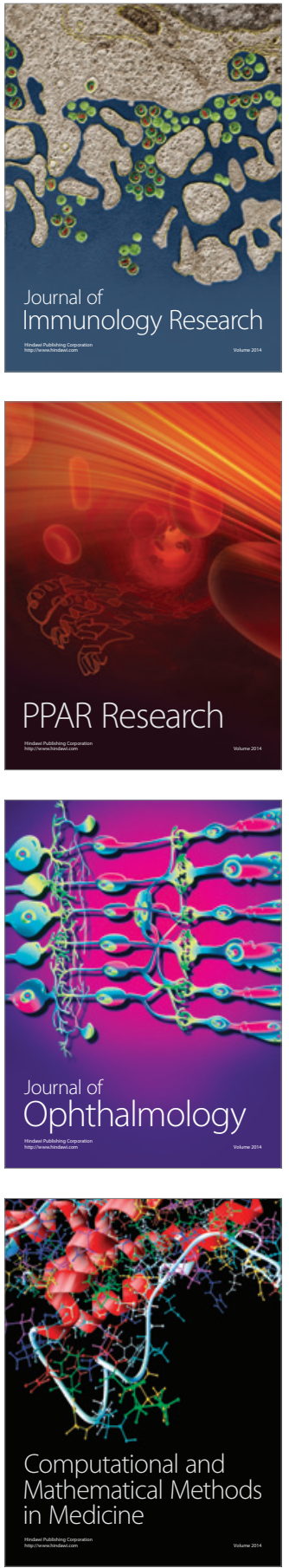

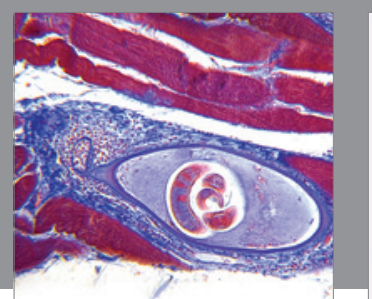

Gastroenterology Research and Practice

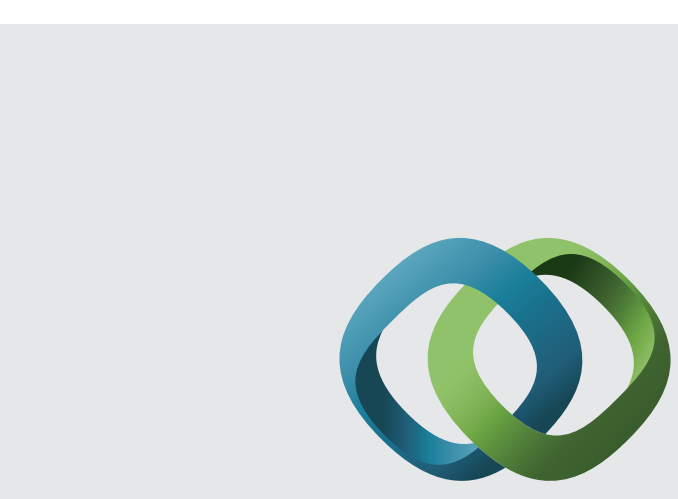

\section{Hindawi}

Submit your manuscripts at

http://www.hindawi.com
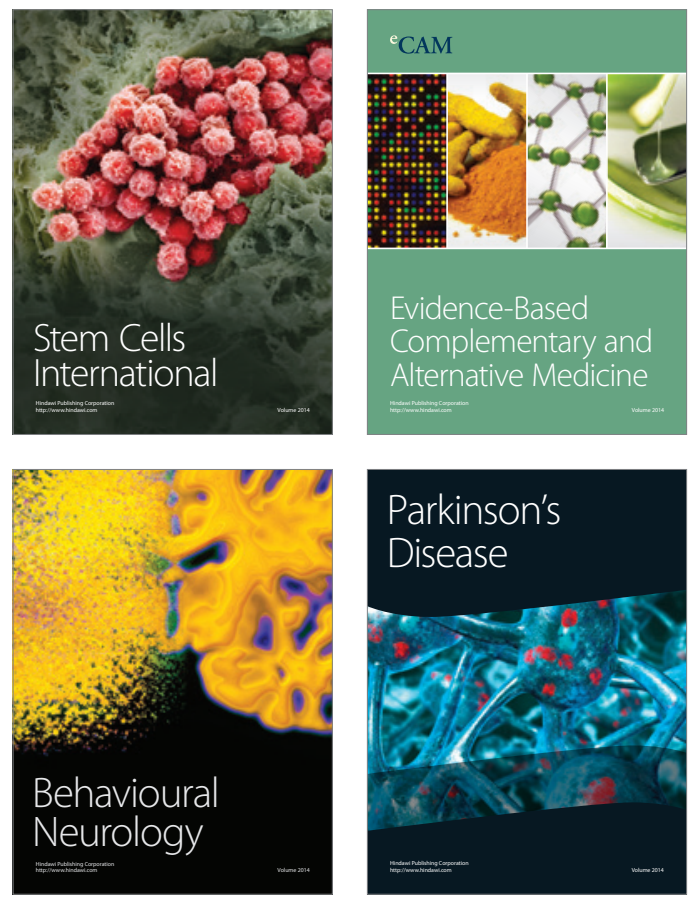
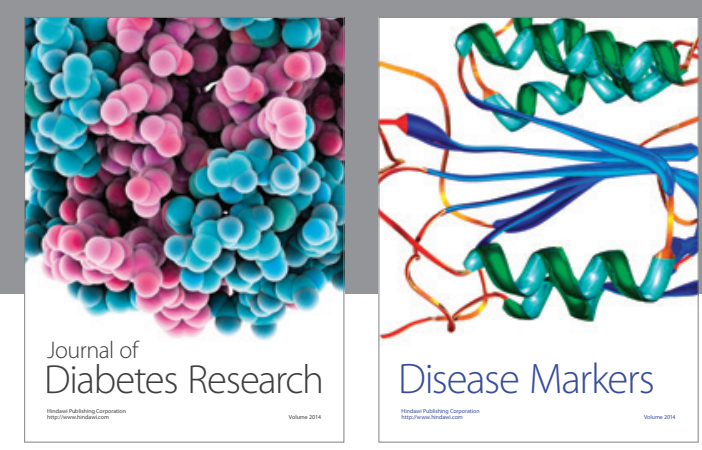

Disease Markers
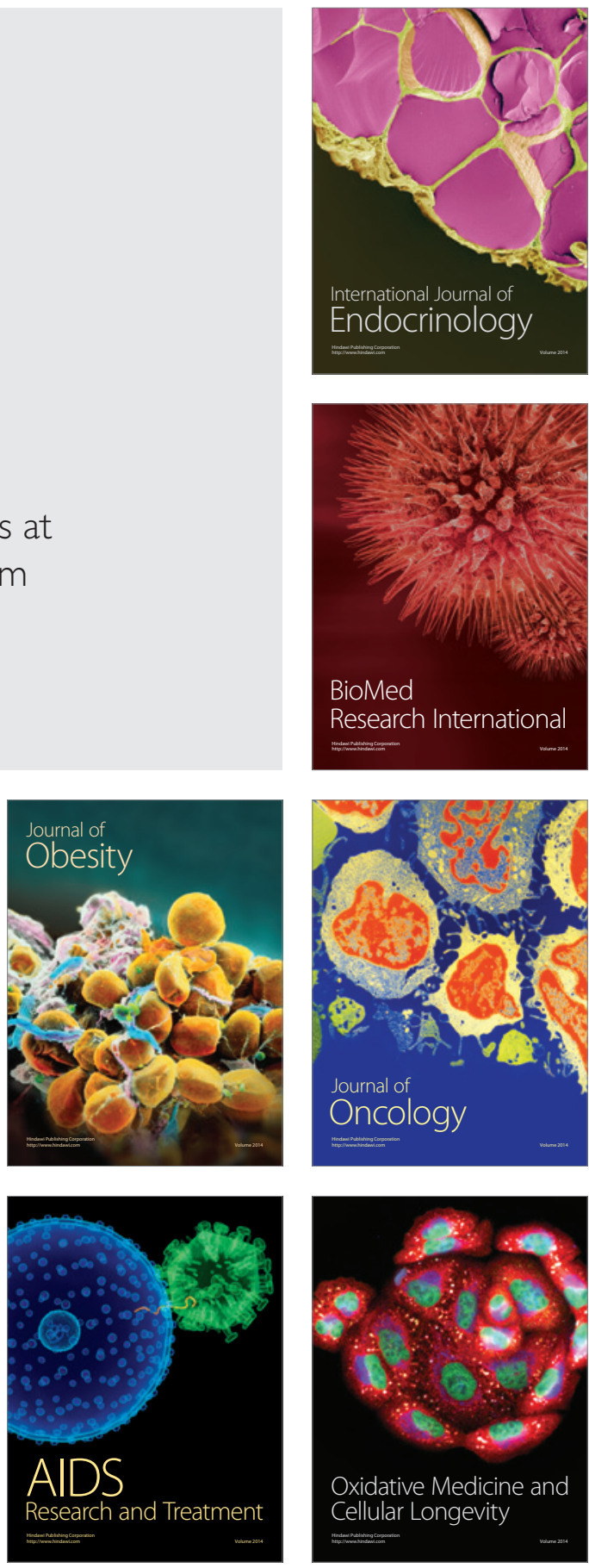